\title{
A Comparison of Heritable Abnormal Lipoprotein Patterns As Defined by Two Different Techniques
}

\author{
D. S. Fredrickson, R. I. LeVy, and F. T. LiNdGren \\ From the Molecular Disease Branch, National Heart Institute, \\ Bethesda, Maryland 20014, and the Donner Laboratory, Lawrence Radiation \\ Laboratory, University of California, Berkeley, California 94720
}

A в S T R A C T Eight plasma lipoprotein patterns currently employed in attempts to identify different forms of familial dyslipoproteinemia have been compared by two methods. The first (NIH method) is based on paper electrophoretic patterns produced by the four lipoprotein classes obtained by paper electrophoresis in albuminated buffer, coupled with the measurement of the total cholesterol of three of these lipoprotein classes and ascertainment of abnormal flotation of beta-migrating lipoproteins. The second (Donner method) is based on lipoprotein patterns obtained in the analytical ultracentrifuge, adapted for computer analysis and complemented by chemical determination of the concentration of chylomicrons (lipoproteins of $\left.\mathrm{S}_{\mathrm{f}}{ }^{\circ}>400\right)$. Pooled samples representing patients with five types of familial hyperlipoproteinemia and three different forms of inherited lipoprotein deficiency were separately analyzed. For six of the eight pools, both methods provided a distinctive lipoprotein pattern in terms of changes in one or more variables. For the remaining two pools, type IV hyperlipoproteinemia and the heterozygote for Tangier disease, both methods provided identical but not unique patterns. The results indicate that lipoprotein analyses obtained by either method may be used interconvertibly in further genetic and other clinical studies.

\section{INTRODUCTION}

Plasma lipoprotein patterns provide a more systematic basis for classification and study of hyper-

\footnotetext{
Received for publication 16 January 1968 and in revised form 25 July 1968.
}

lipidemia than can be achieved by lipid determinations alone. The usefulness of this approach has recently been demonstrated in the study of primary familial hyperlipoproteinemia where the distribution of five basic types of patterns is such as to suggest that the patterns are capable of segregating more different phenotypes than had been detected previously (1). For these studies a system for defining lipoprotein patterns was established at the National Heart Institute to permit the rapid screening of large numbers of samples. The basic technique is paper electrophoresis using albuminated buffer (2), to which are added several other measurements that are relatively simple. Occasionally it is also necessary to employ a single separation at $\mathrm{d}=1.006$ in the preparative ultracentrifuge to measure the concentration of beta lipoproteins and determine their relative density (1). ${ }^{1}$

The NIH system was established on the premise that the four lipoprotein bands obtained with electrophoresis, the alpha, beta, and prebeta lipoproteins, and the chylomicrons, are comparable to major lipoprotein classes defined by other techniques. Experiments have demonstrated the relationship of the bands on paper to fractions obtained by preparative ultracentrifugation and further clarified some of their interrelationships and different roles in fat transport (3-5).

There remains to be demonstrated, however, the extent to which the results obtained by this system are interconvertible to data obtained with an optimal method for quantifying and characterizing lipoproteins. This latter method is provided by the analytical ultracentrifuge, as especially adapted at

${ }^{1}$ Levy, R. I., and D. S. Fredrickson. In preparation. 
the Donner Laboratory for analysis by the computer of the low and high density lipoprotein spectra over many $\mathrm{S}_{\mathrm{f}}{ }^{\circ}$ and $\mathrm{F}$ rate increments, respectively (6), and complemented by a procedure for the isolation and quantification of a chylomicron containing fraction (lipoproteins of $\mathrm{S}_{\mathrm{f}}^{\circ}>400$ ) (7).

Our paper describes such a comparison of lipoprotein analyses obtained by the NIH and Donner systems on pooled plasma samples representing five different types of familial hyperlipoproteinemia and three different forms of inherited lipoprotein deficiency. For several of these disorders, the studies also represent the first definitive lipoprotein determinations by the analytical ultracentrifuge.

\section{METHODS}

\section{Patients}

The patient donors were selected from families studied by the NIH Molecular Disease Branch. Each patient had been thoroughly examined as an outpatient to exclude any other disease known to cause abnormal lipoprotein patterns, and most had been hospitalized one or more times for intensive study. No patient was receiving medications and all had been encouraged to eat a regular diet for at least $1 \mathrm{wk}$ before sampling. All of the available relatives of the patients were also sampled; and in all instances, except for some of the patients with type III hyperlipoproteinemia (see below), and one patient with type II, the same lipoprotein abnormalities were demonstrated in one or more first-degree relatives.

For four of the eight lipoprotein patterns the combined analyses were performed on a single sample composed of equal aliquots of plasma pooled from two or more patients. Similar pools were analyzed for types II, III, and IV, but other individual samples were also run for purposes of confirmation. The eighth pattern, abetalipoproteinemia, was obtained on plasma from a single patient. The different samples were derived and designated in the following manner.

Type I. This pool represented two brothers, J. P. and P. P., age 35 and 19, respectively, from a previously well-characterized family $(8,9)$ in which a total of three of six siblings have severe hyperlipemia dependent upon intake of dietary fat, low plasma postheparin lipolytic activity (PHLA), hepatosplenomegaly, and recurrent bouts of abdominal pain.

Type II. The pool contained samples from three males, R. U., O. H., and S. V., and one female, J. S., 35, 35,33 , and $35 \mathrm{yr}$, respectively, with the most common form of "familial hypercholesterolemia." In each instance the familial involvement was compatible with the expected dominant transmission of the type II abnormality and with the donor being a heterozygote (1). Each of the donors of the type II pool also had tendon xanthomas. Their beta lipoprotein concentrations as individually de- termined varied from 320 to $380 \mathrm{mg} / 100 \mathrm{ml}$ of lipoprotein total cholesterol. Although far above normal, this concentration is well below the lowest value of $650 \mathrm{mg} / 100$ $\mathrm{ml}$ obtained in samples from eight untreated presumed homozygotes for type II examined recently at the NIH.1 Samples were also analyzed from two other patients with type II, who had higher glyceride concentrations than the pool. One patient, L. L., a $67 \mathrm{yr}$ old woman, had extensive tendon xanthomas. Her daughter and two grandchildren also had type II patterns but with normal glycerides. The second patient, F. J., was a $45 \mathrm{yr}$ old male, who had no xanthomas. His father died at age 42 of ischemic heart disease. His four children and mother were normal.

Type III. The pool was comprised of samples from four adults, two males, G. G. and E. A., and two females, M. $L$ and B. R., ages $53,46,56$, and 46 , respectively. Individual samples were also analyzed from J. T., age 39 (two samples taken 30 months apart), his brother Ja. T., age 31, C. H., age 51 , and J. R., age 46 . Seven of these eight type III patients had superficial palmar xanthomas, five also had Achilles tendon xanthomas. Each had been followed for months and observed on multiple occasions to have the same lipoprotein pattern while eating a regular diet. When fed diets that caused weight loss, or that were low in cholesterol, and relatively high in content of polyunsaturated fats, each had a marked reduction in lipoprotein concentrations $(1,10)$. A total of 33 siblings, parents, and children of these eight patients was sampled. Only one of these other relatives had a type III pattern. All of these findings were compatible with the usual clinical syndrome and the apparent "recessive" inheritance and late expression associated with the type III "genotype" (1). Only 11 of the 35 propositi with this pattern studied at NIH to date have had a parent or sibling with the same abnormality. Most of the samples of type III in this study thus represented only presumed phenotypes of the familial abnormality.

Type IV. The pool for this pattern of hyperlipoproteinemia was represented by two males, J. B. and M. K., ages 56 and $35 \mathrm{yr}$. Both were asymptomatic but had moderate obesity and mild diabetes not requiring insulin. The two sons of J. B. had type IV patterns as did the mother and brother of M. K. A sample from S. K., age 56, the mother of M. K., was analyzed separately.

Type $V$. This pool contained samples from two males, J. B. and J. V., and one female, F. J., whose respective ages were 33,24 , and $28 \mathrm{yr}$. All had been originally detected because of recurrent bouts of abdominal pain. The men had normal PHLA; it was not measured in F. J. Each had more than one parent or sibling with a type IV or type $\mathrm{V}$ lipoprotein pattern. For example, F. J. was one of six siblings, two of which had type IV patterns, two others were type $\mathrm{V}$, while the sixth was normal. Both parents had type IV patterns. The admixture of type IV and type $\mathrm{V}$ patterns and the high frequency of familial involvement were compatible with other kindreds of type $\mathrm{V}$ propositi studied thus far (1).

Homozygotes for Tangier disease. The donors, C. No., a $40 \mathrm{yr}$ old male, T. La. and F. La., 10 and $11 \mathrm{yr}$ old 
brother and sister, and Pe. Lo., a girl age 12, have been previously reported in detail (11). They represent typical homozygotes from three of the five reported kindreds with familial alpha lipoprotein deficiency or Tangier disease.

Heterozygotes for Tangier disease. This pool was composed of samples from G. La. and P. La., 29 and $30 \mathrm{yr}$ olds, parents of T. La. and F. La., two of the abnormal children with Tangier disease included in the preceding pool. Each has been examined annually for $7 \mathrm{yr}$ and has had persistently low alpha lipoprotein values with little or no evidence of tissue storage of cholesteryl esters. This has been demonstrated for other presumed heterozygotes for Tangier disease (11)

Abetalipoproteinemia. This sample was obtained from a single patient, A. C., ${ }^{2}$ age 21 , whose sister also has the disease. They have been the subject of prior reports (12) and have been demonstrated to have no native plasma beta lipoprotein detectable by conventional immunochemical tests (5).

Blood samples were obtained 14-16 hr after the evening meal, while the patients were on a regular diet as previously defined for phenotyping (1). The disodium salt of ethylenediaminetetraacetic acid (EDTA), $1 \mathrm{mg} /$ $\mathrm{ml}$ of blood, was used as anticoagulant. Plasma was separated at $4^{\circ} \mathrm{C}$ and the samples kept at these temperatures from 1 to 3 wk before completion of electrophoretic, blood lipid, and lipoprotein analyses. Equal quantities of plasma from each patient were pooled. The pools were divided into two parts, for analyses at the NIH and Donner laboratories, respectively. The latter was shipped by air in containers with wet ice. The refractive index of each pool was measured prior to and on receipt of the shipments. These were unchanged, excluding any dilution or gross contamination during shipment.

\section{Controls}

Clinically healthy employees of the Lawrence Radiation Laboratory, Livermore, Calif., were used as controls for the lipoprotein measurements by the Donner system. Nonfasting morning serum samples were obtained from 16 males and 16 females, ages $35-50 \mathrm{yr} .25 \mathrm{ml}$ of blood was drawn into evacuated tubes containing $1.5 \mathrm{mg}$ of dried Merthiolate, giving a serum concentration of approximately $1 / 10,000$.

$\mathrm{NIH}$ normal values (Table I $a$ ) were obtained from groups of fasting subjects who were clinically healthy and whose plasma triglycerides were $<200 \mathrm{mg} / 100 \mathrm{ml}$ (1).

\section{NIH method}

Plasma cholesterol and triglycerides were determined with the Technicon AutoAnalyzer (13a and b). 5-ml samples of plasma were diluted with $1.5 \mathrm{ml}$ of $0.15 \mathrm{M}$ $\mathrm{NaCl}$ solution and ultracentrifuged at $14^{\circ} \mathrm{C}$ for $16 \mathrm{hr}$ at $100,000 \mathrm{~g}(14)$ in a Spinco 40.3 rotor. The supernatant $(\mathrm{d}<1.006)$ and infranatant $(\mathrm{d}>1.006)$ fractions were

\footnotetext{
2 Courtesy of Dr. Leonard L. Laster.
}

collected and the volume of the infranatant fraction returned to $5-\mathrm{ml}$ by the addition of $0.15 \mathrm{M}$ saline solution. The electrophoretic patterns of both fractions and that of the parent plasma were determined simultaneously with a modification (1) of the method of Lees and Hatch (2). $0.02 \mathrm{ml}$ of plasma was loaded on the strip in each case. The lipoprotein bands were stained with oil-red-O. The particles and lipoproteins containing beta lipoprotein were precipitated from a $3 \mathrm{ml}$ aliquot of plasma by addition of $0.15 \mathrm{ml}$ of $1.0 \mathrm{M}$ manganese chloride and $6 \mathrm{mg}$ of sodium heparin (15). The cholesterol contents of the supernatant from this procedure and the fractions obtained in the preparative ultracentrifuge were then determined. Lipoprotein total cholesterol (Table I $a$ ) was calculated as follows: alpha= cholesterol in the supernatant following precipitation; beta $=$ cholesterol in the fraction of $\mathrm{d}>1.006$ less the alpha cholesterol; very low density lipoproteins (VLDL) = plasma cholesterol less the cholesterol in the fraction of $\mathrm{d}>1.006$. Concentrations were expressed as $\mathrm{mg} / 100 \mathrm{ml}$ of plasma. All cholesterol and triglyceride determinations were done in duplicate.

\section{Donner method}

Lipoprotein isolation and analyses of individual serum samples and the plasma pools were patterned after de Lalla and Gofman (16) and are described in detail elsewhere (6). This procedure utilizes a computer technique for calculating concentrations within $29 \mathrm{~S}_{\mathrm{f}}^{\circ} 0-400$ low density lipoprotein concentration intervals as well as a breakdown of the $F_{1.20} 3.5-9$ and $F_{1.20} 0-3.5$ high density lipoprotein (HDL) concentrations that correspond roughly to the $\mathrm{HDL}_{2}$ and $\mathrm{HDL}_{3}$ classes, respectively. The high density lipoproteins are isolated and analyzed in a $\mathrm{NaBr}$ medium of $\mathrm{d}=1.200$. Moving boundary $\mathrm{S}_{\mathrm{e}}{ }^{\circ}$ rates of the major $\mathrm{S}_{\mathrm{f}}{ }^{\circ}$ 0-12 lipoprotein classes were also calculated by a computer technique (17). Isolation of a chylomicron-containing fraction (lipoproteins of $\mathrm{S}_{\mathbf{t}}{ }^{\circ}>$ 400) was achieved on a nonlinear salt gradient in a swinging bucket rotor with quantification by $\mathrm{N}, \mathrm{C}$, and $\mathrm{H}$ elemental analyses (7).

\section{RESULTS AND DISCUSSION}

The lipoprotein patterns obtained on each pool by the NIH and Donner methods are graphically compared in Figs. 1-5 and 7-10. Quantitative analyses and several important qualitative features of these patterns are presented in Tables I $a$ and I $b$. The data from the Donner system displayed in Table $I b$ and elsewhere are limited to 10 variables which include summed components obtained within prescribed flotation rate intervals. These are sufficient to define the quantitative features on which rest the differences in lipoprotein profiles obtained. Actually, the graphic plots shown in the figures are derived from concentrations calculated 
TABLE I $a$

Plasma Lipids and NIH Values for Lipoproteins

\begin{tabular}{|c|c|c|c|c|c|c|c|}
\hline \multirow[b]{2}{*}{ Sample } & \multirow[b]{2}{*}{ Plasma cholesterol } & \multirow[b]{2}{*}{ Plasma glyceride } & \multicolumn{3}{|c|}{ Lipoprotein cholesterol } & \multicolumn{2}{|c|}{$\begin{array}{l}\text { Presence of } \\
\text { Chylo- }\end{array}$} \\
\hline & & & $\alpha$ & $\beta$ & VLDL* & Floating & band \\
\hline & & & $m g / 100 m l$ & & & & \\
\hline 1 Normal (females) & $\begin{array}{l}217 \pm 35 \ddagger \\
224 \pm 26 \$\end{array}$ & $\begin{array}{r}80 \pm 42 \ddagger \\
103 \pm 47 \S\end{array}$ & $62 \pm 14 \ddagger$ & $130 \pm 24 \ddagger$ & $14 \pm 9 \ddagger$ & $0 \ddagger$ & $0 \ddagger$ \\
\hline 2 Normal (males) & $\begin{array}{l}230 \pm 55 \ddagger \\
263 \pm 49 \S\end{array}$ & $\begin{array}{r}90 \pm 41 \ddagger \\
173 \pm 98 \S\end{array}$ & $49 \pm 10 \ddagger$ & $128 \pm 28 \ddagger$ & $21 \pm 9 \ddagger$ & $0 \ddagger$ & $0 \ddagger$ \\
\hline 3 Type I pool & 300 & 2516 & 15 & 21 & 264 & 0 & + \\
\hline 4 Type II pool & 418 & 123 & 34 & 366 & 18 & 0 & 0 \\
\hline 5 Type III pool & 318 & 179 & 58 & 146 & 114 & + & 0 \\
\hline 6 Type IV pool & 264 & 409 & 32 & 119 & 113 & 0 & 0 \\
\hline 7 Type V pool & 272 & 844 & 20 & 69 & 183 & 0 & + \\
\hline 8 Tangier homozygote pool & 62 & 178 & 2 & 27 & 33 & + & 0 \\
\hline 9 Tangier heterozygote pool & 144 & 213 & 26 & 72 & 46 & 0 & 0 \\
\hline 10 Abetalipoproteinemia & 38 & 5 & 37 & 1 & $\mathbf{0}$ & 0 & 0 \\
\hline
\end{tabular}

* VLDL, all cholesterol in the lipoproteins of $d<1.006$.

$\ddagger$ NIH data, for fasting subjects $40-49 \mathrm{yr}$, plasma values (mean $\pm \mathrm{SD}$ ), 44 female and 67 male subjects (except for $\beta$-lipoprotein cholesterol values, where 30 and 23 subjects, respectively, were included).

$\S$ Donner data, for nonfasting subjects, 35-50 yr, serum values (mean \pm SD) 16 subjects.

TABLE I $b$

Plasma or Serum Lipoprotein Concentrations, Donner Ultracentrifugal Lipoprotein Values

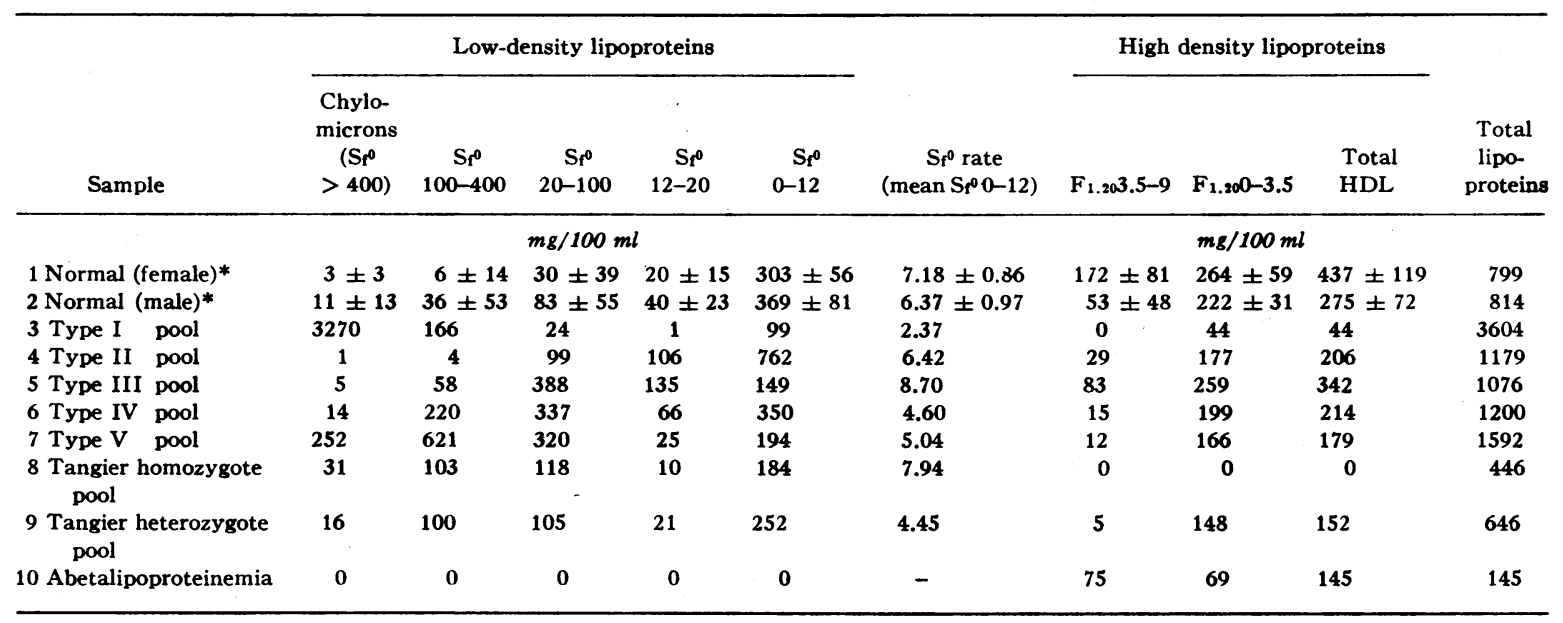

* Serum from 16 patients 35-50 yr, mean \pm SD. All other values are means of duplicate plasma analyses.

by the computer at the following number of flotation rate intervals for each class: $\mathrm{S}_{\mathrm{f}}^{\circ} 0-12,11 ; \mathrm{S}_{\mathrm{f}}{ }^{\circ}$ $12-20,4 ; \mathrm{S}_{\mathrm{f}}^{\circ} 20-100,8 ; \mathrm{S}_{\mathrm{f}}^{\circ} 100-400,6$; and the two high density components of $F_{1.20} 3.5-9$ and $F_{1.20} 0-3.5$ at 7 and 8 intervals respectively.

The equivalent lipoprotein classes defined by the two systems that may be compared in Tables I $a$ and $\mathrm{I} b$ and the figures are as follows: alpha lipoproteins and total HDL, beta and $\mathrm{S}_{\mathrm{f}}^{\circ} 0-12+$ $\mathrm{S}_{\mathbf{f}}{ }^{\circ} 12-20$ lipoproteins, prebeta and $\mathrm{S}_{\mathbf{f}}{ }^{\circ} 20-100+$
$\mathrm{S}_{\mathrm{f}}^{\circ} 100-400$ lipoproteins, and the two "chylomicron" fractions. The two concentrations given for these equivalent lipoproteins are in different terms. One is expressed as total lipoprotein cholesterol, the other as mass of lipoproteins $/ 100 \mathrm{ml}$ of plasma.

\section{The normal pattern}

In the normal adult (Fig. 1) the electrophoretogram contained alpha, beta, and prebeta lipoprotein bands. In the staining technique employed in the 


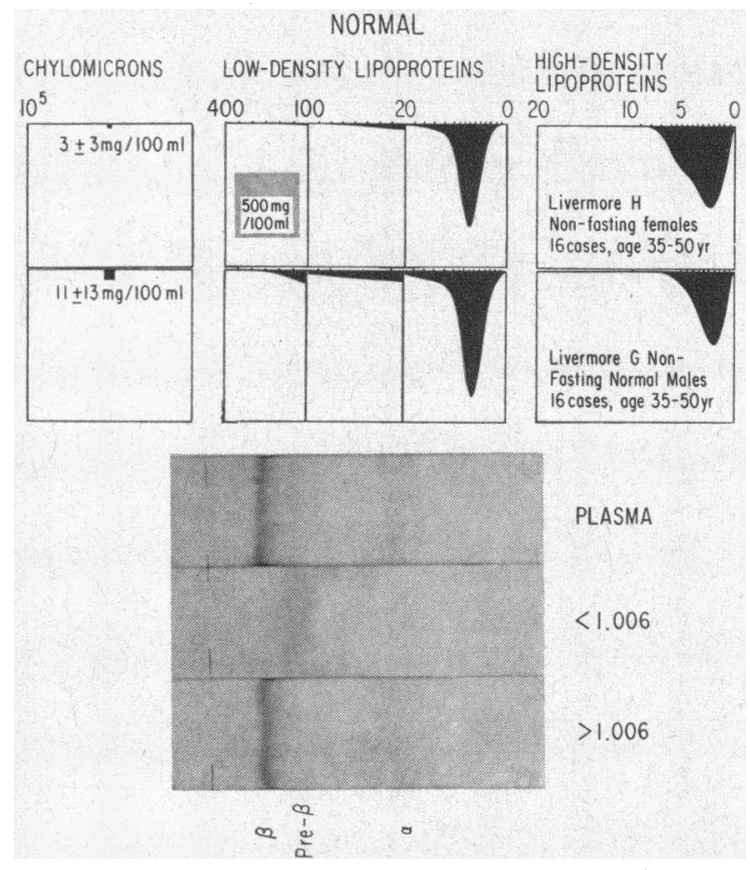

Figure 1 A comparison of normal lipoprotein patterns obtained by the analytical ultracentrifuge combined with chylomicron analyses (above) and electrophoresis (below). The former are derived from pooled data from 16 subjects of each sex (Table I). The latter represents a sample from a 32 yr old male with the following lipid or lipoprotein cholesterol concentrations: plasma cholesterol, 206; alpha, 41; beta, 147; VLDL, 18; and plasma triglyceride, 110 , all in $\mathrm{mg} / 100 \mathrm{ml}$. In this and subsequent figures, the strips represent simultaneous electropherograms of native plasma and the supernatant ("< 1.006") and infranatant ("> 1.006") fractions obtained after preparative ultracentrifugation of plasma as described in the text.

NIH system a faint prebeta band is usually, but not always present; and a chylomicron band is not visible in native plasma obtained after an overnight fast (1). After preparative ultracentrifugation without adjustment of plasma density (a protein- and lipoprotein-free density of approximately $\mathrm{D}=1.006$ ) all lipoproteins of beta mobility in the normal were confined to the infranatant fraction of $\mathrm{d}>1.006$ (Fig. 1).

The lipoproteins of prebeta mobility were all located in the supernatant fraction of $d<1.006$. The intensity of the staining of the prebeta band in Fig. 1 and in subsequent figures was enhanced over that obtained in the whole plasma sample, since the ultracentrifugation results in a twofold increase in concentration of the supernatant frac- tion. The faint trail of dye visible from the origin to the prebeta region in the $\mathrm{d}<1.006$ electrophoretogram shown in Fig. 1 has been considered to represent lipoproteins of $S_{f}^{\circ} 100-400$ or greater (3).

The features of the normal profiles obtained in the analytical ultracentrifuge (Fig. 1) have been described previously (6). These included a greater total concentration and asymmetry in distribution of HDL in females and higher concentrations in males of each of the major classes of low density lipoproteins. Also, the $S_{f}{ }^{\circ}$ rate of the $S_{f}{ }^{\circ} 0-12$ component was higher in females than in males. The low chylomicron concentrations of 3 and 11 $\mathrm{mg} / 100 \mathrm{ml}$ may have been slightly higher in these nonfasting subjects than would be obtained in prebreakfast samples.

\section{Abnormal patterns}

The relative concentrations of the equivalent lipoprotein classes departed from the normal in a comparable fashion in each of the different abnormal pools (Table I). These abnormalities, whether observed on the electrophoretic strips or in the ultracentrifuge, resulted in a distinctive pattern for each of the eight samples.

Types $I, I V$, and $V$. The lipoprotein patterns identified as Types I, IV, and V may conveniently be considered together. These three patterns are associated with hyperglyceridemia (hyperlipemia) that appears to be due to different metabolic abnormalities and their distinction is useful for genetic and other clinical purposes (1).

The three electrophoretic patterns (Figs. 2-4) had the qualitative differences earlier used to define them (1). The type I pattern (Fig. 2), characteristic of familial hyperchylomicronemia, was dominated by a dense chylomicron band. Only very faint beta, prebeta, and alpha lipoprotein bands were visible in keeping with the quantities of cholesterol representing each (Table I $a$ ).

In the type IV pool (Fig. 3) the prebeta lipoprotein band was increased and no chylomicron band was present. This pattern is representative of an excess of glycerides primarily of endogenous origin $(1,3)$. The individual sample from $\mathrm{S}$. K., whose plasma triglyceride was $520 \mathrm{mg} / 100 \mathrm{ml}$, had comparable lipoprotein profiles to the type IV pool. The type V pattern in Fig. 4 contained both a chylomicron band and increased prebeta lipopro- 


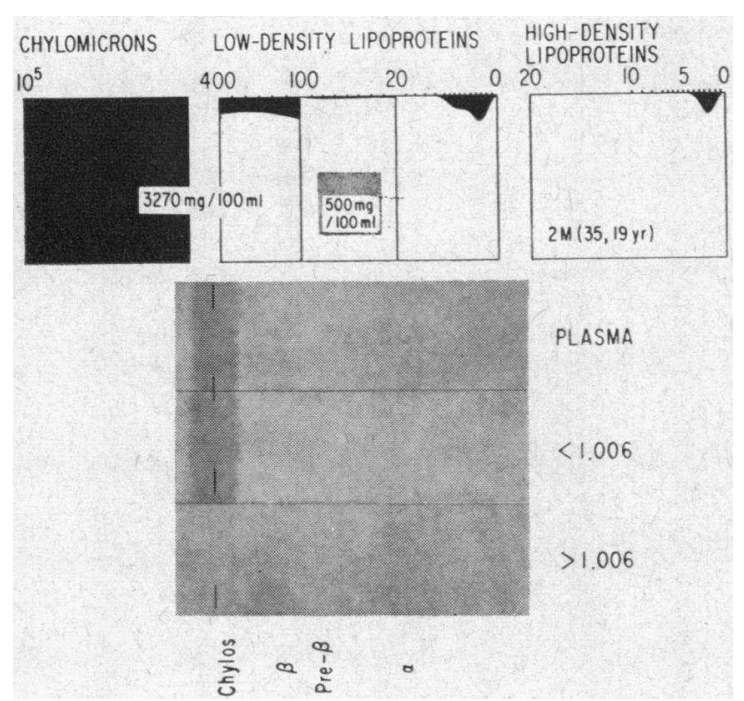

FIGURE 2 Type I hyperlipoproteinemia.

teins, from which it has been inferred that metabolism of both endogenous and exogenous glycerides are disturbed (1). The plasma triglyceride concentrations in the type IV and V pools were elevated to a lesser extent than in the sample of type I and changes in the lipoprotein fractions, in terms of their cholesterol content, corresponded to the electrophoretogram (Table I).

The lipoprotein profiles and chylomicron assays provided by the Donner methods for the type
I, IV, and V pools (Figs. 2-4) were also distinctive and revealed certain quantitative information not heretofore described for these particular lipoprotein abnormalities. In type I the massive quantities of chylomicrons were accompanied by a much smaller increase in $\mathrm{S}_{\mathrm{f}}{ }^{\circ} 100-400$ lipoproteins spread fairly evenly throughout this flotation rate interval.

As predictable from the electrophoretogram, the HDL and $S_{f}^{\circ}$ 0-20 lipoproteins were quite reduced in type $I$; the distribution of the latter was also unusual. There was a suggestion of heterogenity in the distribution of $\mathrm{S}_{\mathrm{f}}^{\circ}$ 0-12 lipoproteins, although the peak of this component at $\mathrm{S}_{\mathrm{f}}^{\circ} 5-8$, was approximately in the normal range.

The profiles for types IV and V had different features, some of them also predictable from the electrophoretogram. In type V, the concentration of chylomicrons was greatly elevated but, in contrast to type $I$, there were also great increases in both $\mathrm{S}_{\mathrm{f}}^{\circ} \quad 20-100$ and $\mathrm{S}_{\mathrm{f}}^{\circ} 100-400$ lipoproteins. These lipoproteins reached maximum concentration within the interval $\mathrm{S}_{f}^{\circ} 60-200$ and were still abundant at the $S_{f}{ }^{\circ} 400$ Schlieren cut-off point (Fig. 4). In the type IV pool the concentration of both $S_{f}^{\circ} 20-100$ and $S_{f}^{\circ} 100-400$ lipoproteins were also increased. The concentration profile of the $S_{f}{ }^{\circ} 20-100$ class was relatively flat compared to a gradual rise with $\mathrm{S}_{\mathrm{f}}{ }^{\circ}$ seen in type V. The

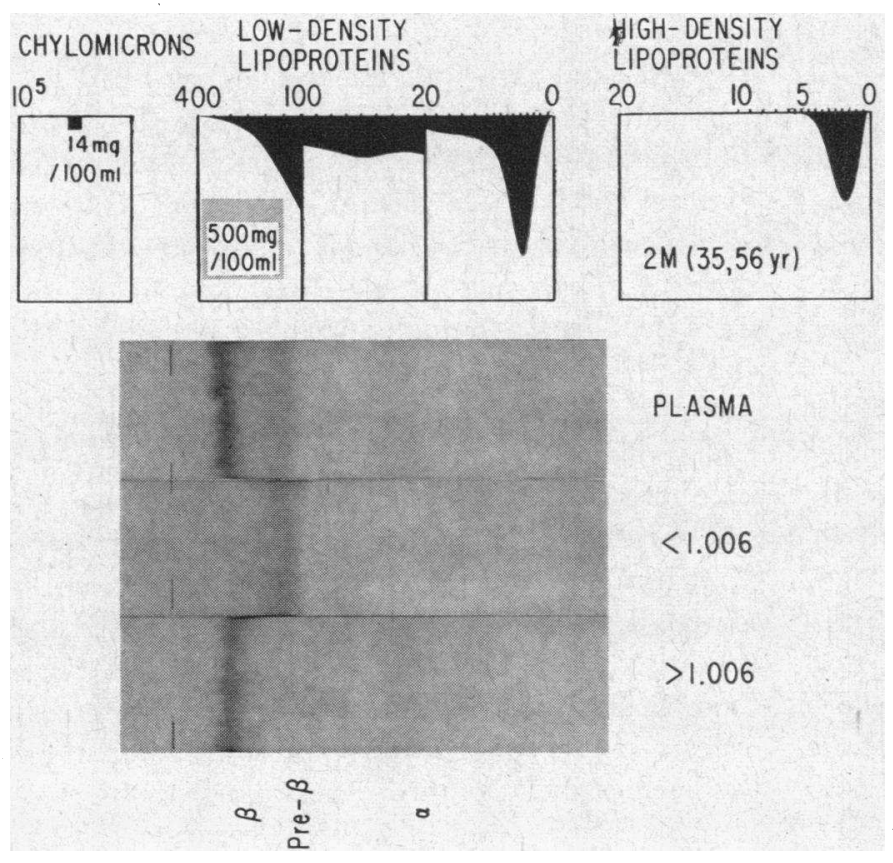

FIGURE 3 Type IV hyperlipoproteinemia. 


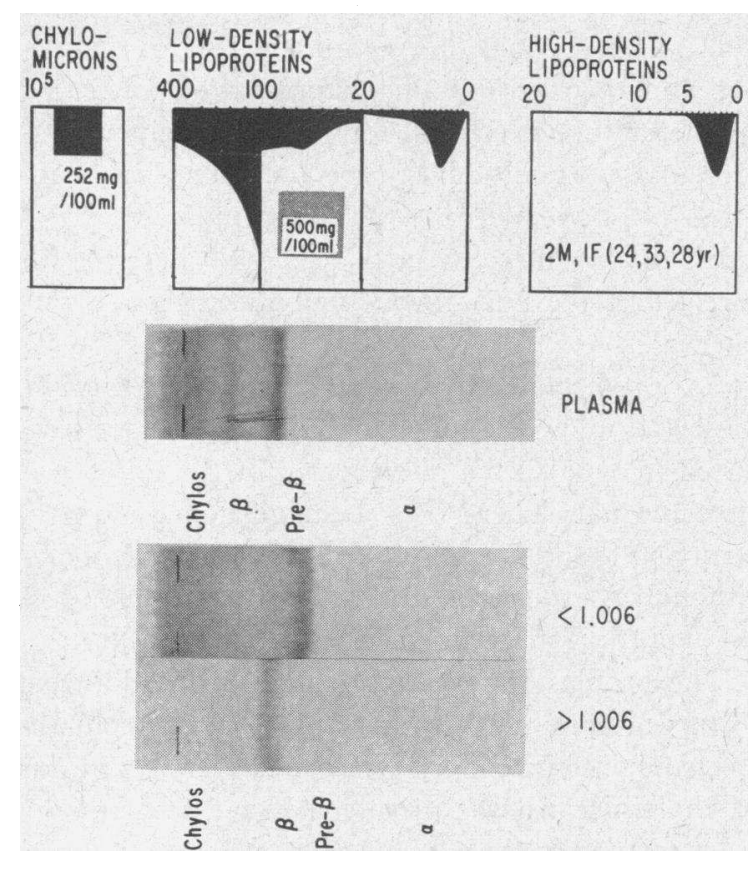

FIgURE 4 Type V hyperlipoproteinemia.

major differences were the return to base line of the $S_{f}{ }^{\circ}>100$ class by $S_{f}{ }^{\circ} 400$ and the normal concentration of chylomicrons in type IV (Fig. 3 and Table I).

In both types IV and $\mathrm{V}$ the concentrations of high density lipoproteins were modestly decreased, and in type $\mathrm{V}$ the concentration of $\mathrm{S}_{\mathrm{f}}{ }^{\circ} 0-20$ was lowered. The mean $S_{f}{ }^{\circ}$ value of the $S_{f}{ }^{\circ} 0-20$ lipoproteins was also significantly decreased, although less markedly' than in type I (Table I $b$ ). The high density lipoproteins in each of types I, $I V$, and $V$ were notable for a proportionately greater decrease in the $\mathrm{F}_{1.20} 3.5-9\left(\mathrm{HDL}_{2}\right)$ fraction (Table $\mathrm{I} b$ ). It was below resolution in type $\mathrm{I}$ in which $\mathrm{F}_{1.20}$ 0-3.5 $\left(\mathrm{HDL}_{3}\right)$ was also profoundly depressed. $\mathrm{HDL}_{3}$ was not definitely abnor$\mathrm{mal}$ in types IV and V.

Type II. In the pooled type II sample the beta lipoprotein band was sharp and intensely stained (Fig. 5) and the beta lipoprotein cholesterol increased threefold (Table I). The prebeta band appeared to be of normal intensity and the VLDL total cholesterol was not significantly increased (Table I $a$ ). The striking profile in the analytical ultracentrifuge (Fig. 5) was dominated by a sharp peak in the elevated $\mathrm{S}_{\mathrm{f}}{ }^{\circ} 0-12$ class; however, the $\mathrm{S}_{\mathrm{f}}{ }^{\circ}$ rate of 6.4 appeared normal. The $\mathrm{S}_{\mathrm{f}}{ }^{\circ} 12-20$ class was increased roughly to the same extent.

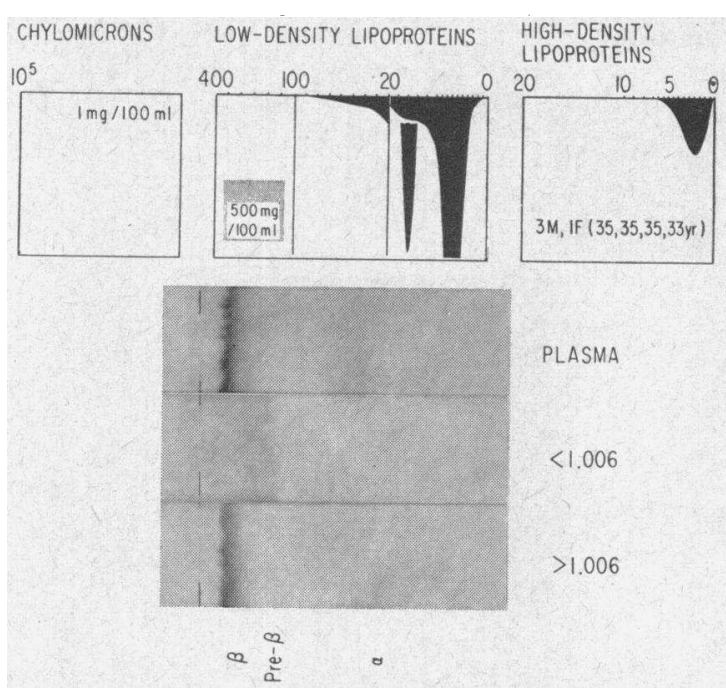

Figure 5 Type II hyperlipoproteinemia. Note that the single sharp beta lipoprotein peak between $S_{*}$ 0-20 has been broken into two parts for ease of illustration.

Since this pool consisted of samples from three males and one female, the concentrations of the $\mathrm{S}_{\mathrm{f}}^{\circ}$ 20-100 class were not considered to be definitely abnormal (Table I $a$ ).

Type II patients sometimes have abnormally high plasma glycerides. The mean of 200 patients at $\mathrm{NIH}$ is approximately $150 \mathrm{mg} / 100 \mathrm{ml}$, the range from 30 to 870 . Profiles on two such hyperglyceridemic patients are shown in Fig. 6. Patient L. L. has the highest glyceride ever seen by us in type II $(870 \mathrm{mg} / 100 \mathrm{ml})$, even though her affected child and grandchildren have "pure" hyperbetalipoproteinemia with normal glycerides. Both profiles in Fig. 6 show the expected marked increase in $\mathrm{S}_{\mathrm{f}}^{\circ}$ 0-12 and 12-20 lipoproteins, but also elevations in $\mathrm{S}_{f}^{\circ} 20-100$ and $100-400$ lipoproteins. Chylomicrons were normal. All of the increased beta lipoprotein was of $d>1.006$. Increased concentrations of very low density lipoproteins have previously been reported in patients having manifestations consonant with the disorder here referred to as type II $(18,19)$.

Type III. Comparison of the pattern in type III with that of type II was of particular interest, for recent studies (1) appear to have proved the validity of an earlier suggestion $(18,20)$ that these lipoprotein patterns represent different disorders. In the NIH system the type III pattern has been defined as a broad beta band on the electrophoretogram coupled with the separate demon- 


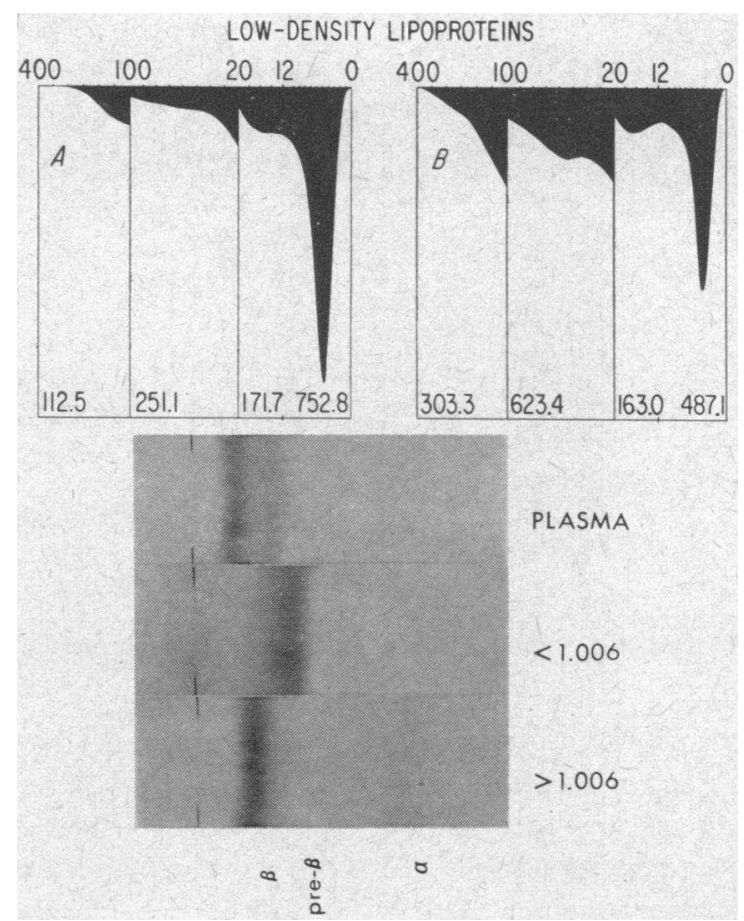

FIGURE 6 Lipoprotein patterns in two patients with type II hyperlipoproteinemia and elevated plasma glycerides. A. Pattern from F. J. whose plasma cholesterol was 390 $\mathrm{mg} / 100 \mathrm{ml}$, triglycerides 332 ; VLDL, beta and alpha lipoprotein cholesterols were 61,298 , and 33 , respectively. B. Pattern from L. L. whose plasma cholesterol was 439 , and triglycerides 870 . The lipoprotein concentrations by the Donner method, in $\mathrm{mg} / 100 \mathrm{ml}$, appear below the profiles. The electrophoretogram is that of $F$. J. In neither patient were $\beta$-migrating lipoproteins present in the $\mathrm{d}<$ 1.006 fraction.

stration of the presence of beta-migrating lipoproteins in the ultracentrifugal supernatant fraction of $\mathrm{d}<1.006(1)$. These qualitative features

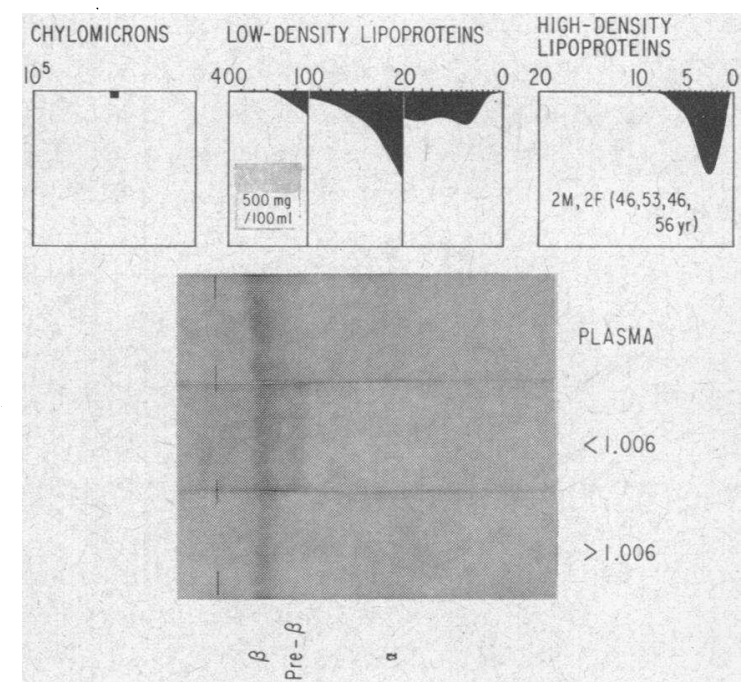

FIGURE 7 Type III hyperlipoproteinemia.

were clearly demonstrated in the type III pool shown in Fig. 7 and are unique for type III, with the exception of the Tangier homozygote (see below).

The pools for types II and III were designed such that the plasma glyceride concentrations would be fairly similar and thus relatively low for type III, in which glycerides usually range from $200-1000 \mathrm{mg} / 100 \mathrm{ml}$ (mean of 48 patients, 475 $\mathrm{mg} / 100 \mathrm{ml}$ ). Five analyses from more representative type III patients with glycerides from 380 to 534 are summarized in Table II. All of these patients have beta lipoproteins that float at $\mathrm{d}<1.006$. In these patients and the pool, the beta lipoprotein cholesterol was low or normal and that in VLDL abnormally high.

In the analytical ultracentrifuge the "broad beta"

TABLE II

Plasma Lipids and Lipoproteins in Individual Type III Patients

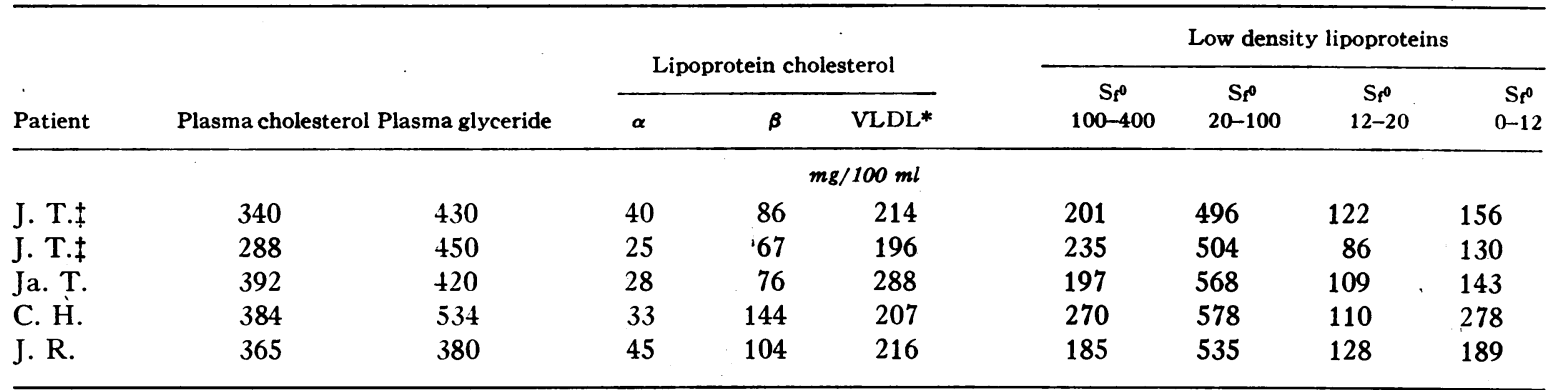

* VLDL, all lipoproteins of D $<1.006$.

$\ddagger$ Analyses of samples separated by a 30 month interval. 
band on the pherogram was approximated by an anomalous disproportion between $\mathrm{S}_{\mathrm{f}}{ }^{\circ} 0-12$ and 12-20 lipoprotein concentrations (Fig. 7; Tables I and II). The $S_{f}^{\circ}$ 0-12 lipoproteins were low or low normal in concentration, their mean $\mathrm{S}_{\mathrm{f}}{ }^{\circ}$ rate $\left(\mathrm{S}_{\mathrm{f}}{ }^{\circ} 8.7\right.$ in the pool, Fig. 7$)$ possibly abnormally high. A relative and absolute increase in $\mathrm{S}_{\mathrm{f}}^{\circ}$ 12-20 lipoproteins continued into the $\mathrm{S}_{\mathrm{f}}{ }^{\circ} 20$ 100 class. In the pool the concentration of the $S_{f}{ }^{\circ}$ 12-100 class was roughly 5-10 times normal ( $\mathrm{Ta}$ ble $\mathrm{I} b$ ), and the peak concentration shifted abnormally upward to about $\mathrm{S}_{\mathrm{f}}^{\circ} 17$ (Fig. 7). The same general changes occurred in the type III patients with higher glycerides, but in them there were also greater increases in $\mathrm{S}_{\mathrm{f}}{ }^{\circ} \quad 20-100$ and 100-400 lipoproteins (Table II). Most of these patients had not returned to baseline by $\mathrm{S}_{\mathrm{f}}{ }^{\circ} 400$ and small amounts of chylomicrons were present at the origin of their electrophoretograms. Alpha lipoproteins were either low or normal in type III.

The type III pattern could thus be distinguished from type II by either method. It is possible that the type III and type IV patterns might occasionally be difficult to distinguish by the quantitative analyses (Tables I and II), and the most reliable definition of type III would appear to be the demonstration of beta-migrating lipoproteins of $d<$ 1.006.

Tangier disease; homozygous abnormal. The lipoprotein pattern present in this rare phenotype was easily detected by either the NIH or Donner systems (Fig. 8). The electrophoretogram contained no alpha lipoproteins; but, as determined by the precipitation technique, a very small amount of alpha lipoproteins were present (Table I $a$ ). This pool contained the small amounts of immunochemically reactive alpha lipoprotein seen in plasma of all Tangier homozygotes (21).

Consonant with previous demonstrations (4), in the electrophoretogram there was also broadening of the beta band and some of the lipoproteins of beta mobility were abnormally present in the supernatant fraction of $d<1.006$. This abnormal flotation decreased the beta lipoprotein subnatant concentration as determined by the NIH method (Table I $a$ ).

The ultracentrifuge was unable to detect any HDL. The profile of $S_{f}^{\circ} 0-20$ lipoproteins was not remarkable, and, since the mean age of the patients

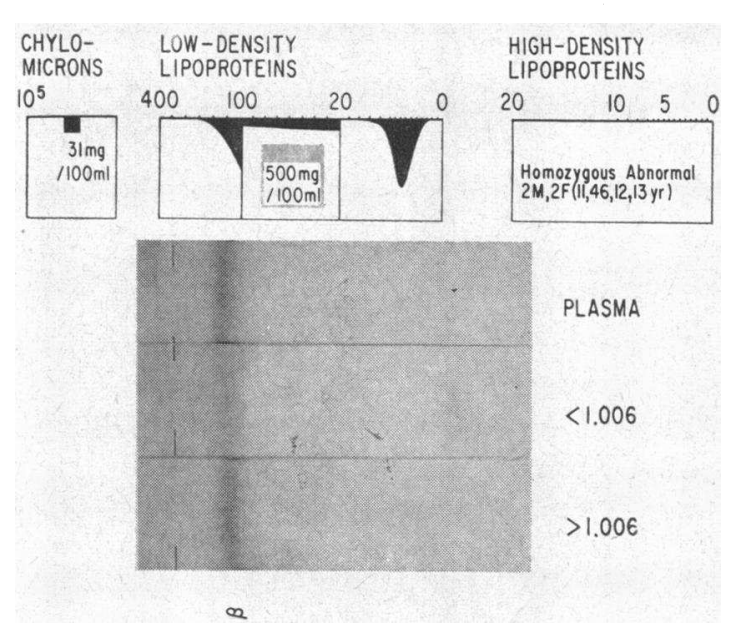

FigUre 8 Tangier disease, homozygous abnormal.

contributing to this pool was considerably less than of the controls, the concentration of $\mathrm{S}_{\mathrm{f}}{ }^{\circ}$ 0-12 lipoproteins and their major $S_{f}^{\circ}$ rate was considered to be normal (Table I $b$ ). On the other hand, the concentrations of $S_{f}{ }^{\circ} 20-100$, and particularly the $\mathrm{S}_{\mathrm{f}}{ }^{\circ} 100-400$ lipoproteins appeared to be abnormally high. The chylomicron fraction was also somewhat elevated.

Abnormalities in the lower density lipoproteins in the Tangier homozygote have been previously noted and a possible minor defect in chylomicron metabolism suspected (11). There is also a partial explanation for what appear to be discrepancies between the ultracentrifugal and electrophoretic patterns of the low density lipoproteins in Tangier disease. Despite an increase in plasma glycerides and of ultracentrifugally defined lipoproteins that normally would be prebeta lipoproteins on electrophoresis, no prebeta band was present (Fig. 7). This phenomenon is attributed to the virtual absence of alpha lipoproteins (4), believed to be responsible normally for the enhanced electrophoretic mobility of the very low density complexes (4). Such alpha lipoprotein-poor complexes thus appear as beta migrating lipoproteins of $d<1.006$, a phenomenon that is distinctly abnormal and shared only with type III hyperlipoproteinemia (Fig. 6). The explanation for this flotation anomaly advanced for Tangier disease is not necessarily applicable to the type III pattern. The plasma concentrations of alpha lipoproteins and HDL are usually normal in this syndrome (Table I), and the presence of alpha lipoproteins in their VLDL 


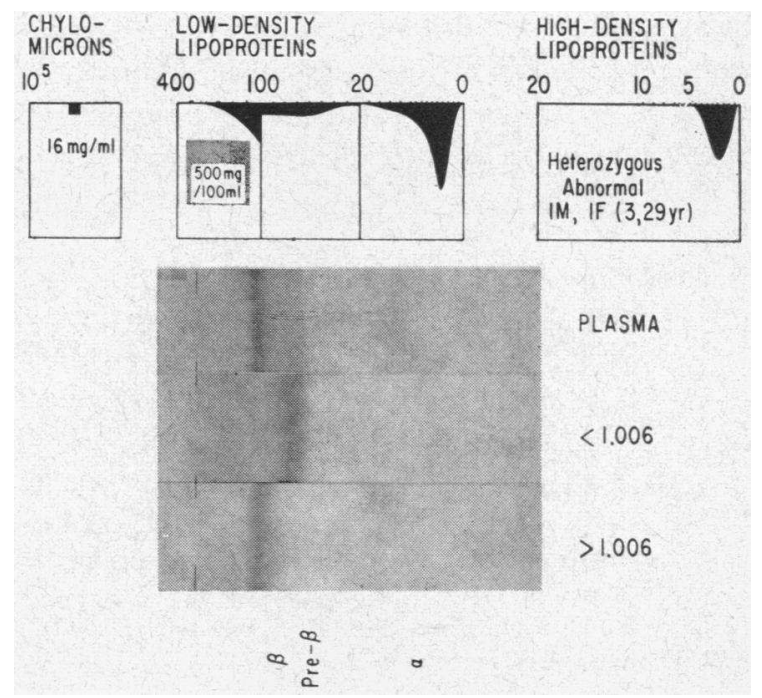

Figure 9 Tangier disease, heterozygous abnormal.

has not yet been quantitatively compared to normal VLDL.

Tangier disease; heterozygous abnormal. $\mathrm{Pa}-$ tients who presumably carry a single abnormal gene for Tangier disease usually have depressed alpha lipoprotein concentrations (11). While the electrophoretogram from this pool (Fig. 9) contained a visible alpha band, the total cholesterol in alpha lipoproteins was abnormally low (Table I a). Plasma glycerides were elevated and accompanied by an appropriate increase in the prebeta band. All of the beta migrating lipoproteins had a d > 1.006 (Fig. 9).

The results in the analytical ultracentrifuge were comparable. The total HDL concentrations were low (Table I $b$ ). The remaining profile (Fig. 9) was similar to that of the homozygote, there being abnormal increases in terms of the $\mathrm{S}_{f}{ }^{\circ}$ 20-100 and $S_{f}^{\circ} 100-400$ lipoproteins. The chylomicrons, although somewhat elevated, were less than the homozygote.

The two methods produced comparable patterns that are distinctive in comparison to the other patterns tested, but the inference is to be avoided that this pattern is phenotypic of the Tangier heterozygote. Although these subjects tend to have increased VLDL associated with their low alpha lipoproteins (11), this combination appears in other abnormalities and may be indistinguishable from familial type IV hyperlipoproteinemia (Table I). If either of the analytical methods de- scribed here are used, the assignment of the heterozygous Tangier phenotype also requires at least the further knowledge that the patient shares blood relationship with a known abnormal homozygote for this disease.

Abetalipoproteinemia. The complete absence of a beta band on electrophoresis and the absence of all lipoproteins of $S_{f}^{\circ}>0$ shown in Fig. 10 describe a unique set of patterns in abetalipoproteinemia. The HDL profile in the analytical ultracentrifuge was also remarkable among all the samples tested, there being a pronounced two-component concentration curve with increased $F$ rate such that it was necessary to analyze the $48^{\prime}$ rather than the standard 64' up-to-speed Schlieren photograph. Somewhat comparable shifts in HDL have been recorded in this disease with the preparative ultracentrifuge $(5,22)$. Although no low density lipoproteins were detected here, small amounts of alpha lipoproteins have been found by immunochemical means in the 1.006-1.063 density range of $\mathrm{S}_{\mathrm{f}}{ }^{\circ}$ 0-20 lipoproteins in all of seven patients examined in one study (5).

\section{General}

The present study was not designed to substantiate the value of a particular plasma lipoprotein pattern for defining a specific phenotype or disease. This still remains to be established for some of these patterns. Nor was this study intended to define adequately the degree of variability of such patterns in one or more patients. What has been achieved is a demonstration of the high degree to

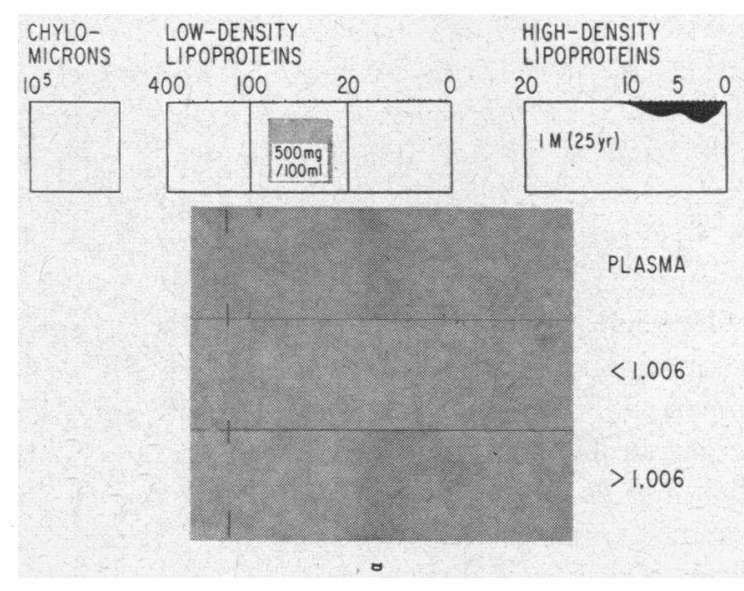

FIGURE 10 Abetalipoproteinemia. 
which data obtained by electrophoresis and the analytical ultracentrifuge are interconvertible.

It is important to point out that comparisons of results from the major methods for defining lipoproteins will likely remain qualitative rather than quantitative. This is true even though some quantification of the electrophoretic strips is possible, either by strip-scanning, elution of bands, or by ancillary procedures such as are used in the $\mathrm{NIH}$ system. In the present experiments there was reasonable correspondence between the concentrations of alpha lipoprotein cholesterol (Table $\mathrm{I} a$ ) and those of total HDL (Table $\mathrm{I} b$ ) in six of the eight plasma pools. The precipitation technique for determining alpha lipoproteins is least reliable when appreciable chylomicrons are present. This probably accounts for the major discrepancies between the different methods as applied to pools from type I and type V. Even the measure of agreement obtained in these studies does not permit the conclusion that lipoproteins having given flotation characteristics and electrophoretic mobility can ever be precisely equated.

These considerations notwithstanding, the present study supports two conclusions of practical importance. The first is that appropriate procedures based on electrophoresis may continue to be used in clinical studies with reasonable confidence that all the gross abnormalities in lipoprotein patterns that are currently known will be detected. This has particularly important implications for genetic studies where screening on a large scale is often necessary both to establish a genotype and to determine its distribution. For this, simple and inexpensive procedures are paramount. The analytical ultracentrifuge has far superior resolution and capacity for quantification. Its potential is thus greater for uncovering subvarieties of major abnormalities and for understanding the metabolic errors involved. The limitations on its application lie in the considerable cost in time and resources required for individual analyses.

Herein lies a second implication of the favorable comparisons between the two lipoprotein techniques. All of the data supplied by each is not interchangeable, but the clinical or genetic abnormalities they may define can be interrelated by common nomenclature, perhaps using simplified terms. The feasibility of this has been demonstrated, and it remains for the appropriate termi- nology to evolve. Such an approach will make possible a translation of results from different laboratories that will much enhance the usefulness of lipoprotein patterns in clinical investigations.

\section{REFERENCES}

1. Fredrickson, D. S., R. I. Levy, and R. S. Lees. 1967. Fat transport in lipoproteins: an integrated approach to mechanisms and disorders. New Engl. J. Med. 276: $32,94,148,215,273$.

2. Lees, R. S., and F. T. Hatch. 1963. Sharper separation of lipoprotein species by paper electrophoresis in albumin-containing buffer. J. Lab. Clin. Med. 61: 518 .

3. Lees, R. S., and D. S. Fredrickson. 1965. Differentiation of exogenous and endogenous hyperlipemia by paper electrophoresis. J. Clin. Invest. 44: 1968.

4. Levy R. I., R. S. Lees, and D. S. Fredrickson. 1966. Nature of pre-beta (very low density) lipoproteins. J. Clin. Invest. $45: 63$.

5. Levy, R. I., D. S. Fredrickson, and L. Laster. 1966. Lipoproteins and lipid transport in abetalipoproteinemia. J. Clin. Invest. 45: 531.

6. Ewing, A. M., N. K. Freeman, and F. T. Lindgren. 1965. The analysis of human serum lipoprotein distributions. In Advances in Lipid Research. R. Paoletti and D. Kritchevsky, editors. Academic Press Inc., New York. 3: 25.

7. Hatch, F. T., N. K. Freeman, L. C. Jensen, G. R. Stevens, and F. T. Lindgren. 1967. Ultracentrifugal isolation of serum chylomicron-containing fractions with quantitation by infrared spectrometry and $\mathrm{NCH}$ elemental analysis. Lipids. 2:183.

8. Havel, R. J., and R. S. Gordon, Jr. 1960. Idiopathic hyperlipemia: metabolic studies in an affected family. J. Clin. Invest. 39: 1777.

9. Fredrickson, D. S., and R. S. Lees. 1966. Familial hyperlipoproteinemia. In The Metabolic Basis of Inherited Disease. J. B. Stanbury, J. B. Wyngaarden, and D. S. Fredrickson, editors. McGraw-Hill Book Co., New York. 2nd edition. 429.

10. Levy, R. I., S. H. Quarfordt, H. R. Sloan, W. V. Brown, and D. S. Fredrickson. 1967. Drug and dietary management of Types II and III hyperlipoproteinemia, two forms of "familial hypercholesterolemia." Circulation. 36: II-171. (Abstr.)

11. Fredrickson, D. S. 1966. Familial high-density lipoprotein deficiency: Tangier disease. In The Metabolic Basis of Inherited Disease. J. B. Stanbury, J. B. Wyngaarden, and D. S. Fredrickson, editors. McGrawHill Book Co., New York. 2nd edition. 476.

12. Farquhar, J. W., and P. Ways. 1966. Abetalipoproteinemia. In The Metabolic Basis of Inherited Disease. J. B. Stanbury, J. B. Wyngaarden, and D. S. Fredrickson, editors. McGraw-Hill Book Co., New York. 2nd edition. 509.

13a. Kessler, G., and H. Lederer. 1966. Fluorometric measurement of triglycerides. In Automation in Analytical Chemistry. Technicon Symposium, 1965. Mediad, Inc., New York. 341. 
b. 1964. Total cholesterol procedure N-24b. In AutoAnalyzer Manual. Chauncey, N. Y., Technicon Instruments.

14. Havel, R. J., H. A. Eder, and J. H. Bragdon. 1955. Distribution and chemical composition of ultracentrifugally separated lipoproteins in human serum. $J$. Clin. Invest. 34 : 1345.

15. Burstein, M., and J. Samaille. 1960. Sur un dosage rapide du cholesterol lié aux $\alpha$ - et aux $\beta$-lipoproteines du sérum. Clin. Chim. Acta. 5: 609.

16. de Lalla, O., and J. Gofman. 1954. Ultracentrifugal analysis of serum lipoproteins. In Methods of Biochemical Analysis. D. Glick, editor. Interscience Publishers, New York. 1: 459.

17. Lindgren, F. T., N. K. Freeman, A. M. Ewing, and L. C. Jensen. 1966. Serum lipoprotein distribution, flotation rates and protein analysis. J. Am. Oil Chemists' Soc. 43: 281.
18. Gofman, J. W., O. de Lalla, F. Glazier, N. K. Freeman, F. T. Lindgren, A. V. Nichols, B. Strisower, and A. R. Tamplin. 1954. The serum lipoprotein transport system in health, metabolic disorders, atherosclerosis and coronary artery disease. Plasma. 2: 413.

19. Guravich, J. L. 1959. Familial hypercholesteremic xanthomatosis : a preliminary report. I. Clinical, electrocardiographic and laboratory considerations. $\mathrm{Am} . \mathrm{J}$. Med. 26: 8 .

20. McGinley, J., H. B. Jones, and J. W. Gofman. 1952. Lipoproteins and xanthomatous diseases. J. Invest. Dermatol. 19 : 71.

21. Levy, R. I., and D. S. Fredrickson. 1966. Nature of alpha lipoproteins in Tangier disease. Circulation. 34 (Suppl. III) : 156. (Abstr.)

22. Jones, J. W., and P. Ways. 1967. Abnormalities of high density lipoproteins in abetalipoproteinemia. $J$. Clin. Invest. 46: 11151. 